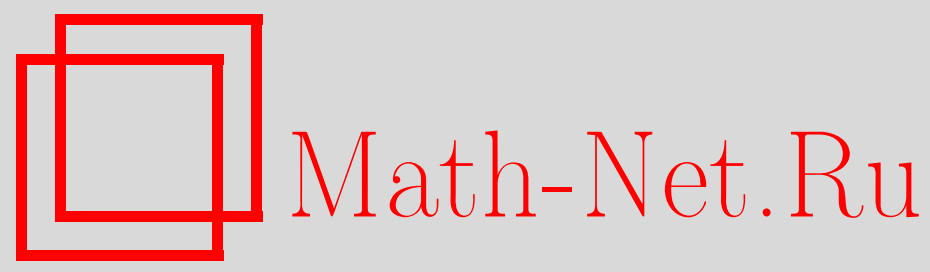

М. А. Чешкова, О паре гиперповерхностей в евклидовом пространстве, Матем. заметки, 2004, том 75, выпуск 3, 474-475

DOI: https://doi.org/10.4213/mzm548

Использование Общероссийского математического портала Math-Net.Ru подразумевает, что вы прочитали и согласны с пользовательским соглашением http://www.mathnet.ru/rus/agreement

Параметры загрузки:

IP : 54.198 .55 .26

26 апреля 2023 г., $14: 32: 47$

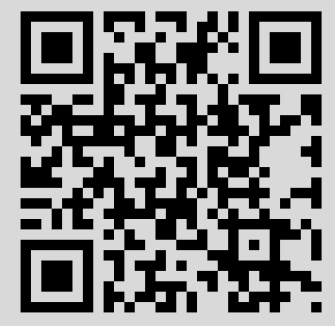




\section{О ПАРЕ ГИПЕРПОВЕРХНОСТЕЙ В ЕВКЛИДОВОМ ПРОСТРАНСТВЕ}

\section{М. А. Чешкова}

Говорят, что отображение $f: M \rightarrow \bar{M}$ для двух римановых многообразий $M, \bar{M}$ сильно сохраняющее кривизну, если $f$ отображает $\nabla^{m} R$ в $\bar{\nabla} m \bar{R}$ для каждого $m=0,1,2, \ldots$, где $\nabla^{m} R, \bar{\nabla} \bar{\nabla}^{m}$ обозначает $m$-е ковариантные производные тензорных полей кривизны $R$ на $M$ и $\bar{R}$ на $\bar{M}$, соответственно [1, с. 324]. Номидзу и Яно [1], [2] доказали теорему: сильно сохраняющ,ий кривизну диффеоморфизм между неприводимыми аналитическими римановыми многообразиями $M u$ $\bar{M}$ размерности $\geqslant 2$ есть гомотетическое преобразование.

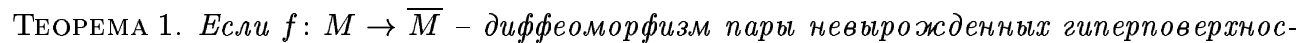
тей в евклидовом пространстве $E^{n}, n>3$, сохраняющий тензоры кривизны, то первые фундаментальные формы $g, \bar{g}$ и вторые фундаментальные формы $b, \bar{b}$ гиперповерхностей $M, \bar{M}$ удовлетворяют равенствам $\bar{g}=t^{2} g, \bar{b}=t b, t \in R$.

Теорема 2. Если $f: M \rightarrow \bar{M}-$ диффеоморфизм пары невырожсденных гиперповерхностей в евклидовом пространстве $E^{n}, n>3$, сохраняющий тензоры кривизны, то локально гиперповерхность $\bar{M}$ получена из гиперповерхности $M$ с помощью гомотетии $u$ изометрии в $E^{n}$.

1. Основные формулы. Пусть $M, \bar{M}$ - две гладкие гиперповерхности в евклидовом пространстве $E^{n}, f: M \rightarrow \bar{M}$ - диффеоморфизм, $F(M)-R$-алгебра дифференцируемых на $M$ функций, $T_{s}^{q}(M)-F$-модуль дифференцируемых на $M$ тензорных полей типа $(q, s), \partial$ - дифференцирование в $E^{n}$.

Формулы Гаусса-Вейнгартена гиперповерхности $M$ имеют вид [1, с. 23]

$$
\begin{aligned}
\partial_{X} Y & =\nabla_{X} Y+b(X, Y) n, \\
\partial_{X} n & =-A X
\end{aligned}
$$

где $X, Y \in T_{0}^{1}(M), \nabla$ - связность Леви-Чивита метрики $g(X, Y)=\langle X, Y\rangle ; b(X, Y)=g(A X, Y)$ вторая фундаментальная форма поверхности $M, A \in T_{1}^{1}(M)$ - оператор Вейнгартена, $n$ - орт нормали, $\langle\cdot, \cdot\rangle$ - скалярное произведение в $E^{n}$. Вьполняются уравнения Гаусса-Кодацци

$$
\begin{gathered}
R(X, Y) Z=b(Y, Z) A X-b(X, Z) A Y \\
\left(\nabla_{Y} b\right)(X, Z)=\left(\nabla_{X} b\right)(Y, Z),
\end{gathered}
$$

где $R(X, Y) Z=\nabla_{X} \nabla_{Y} Z-\nabla_{Y} \nabla_{X} Z-\nabla_{[X, Y]} Z-$ тензор кривизны связности $\nabla,\left(\nabla_{X} b\right)(Y, Z)$ $=X b(Y, Z)-b\left(\nabla_{X} Y, Z\right)-b\left(Y, \nabla_{X} Z\right)-$ ковариантная производная поля $b$ в связности $\nabla$.

Обозначим через $r$ радиус-вектор точки $p \in M$, через $\bar{r}$-радиус-вектор точки $f(p) \in \bar{M}$. Тогда отображение $f: M \rightarrow \bar{M}$ запишется в виде

$$
\bar{r}=f(r)
$$

Дифференциал отображения $f$ определится из равенства $d f(X)=d f\left(\partial_{X} r\right)=\partial_{X} \bar{r}, X \in T M$. Отображение $f$ индуцирует на $M$ метрику

$$
\bar{g}(X, Y)=\langle d f(X), d f(Y)\rangle .
$$

Пусть $\bar{b}^{*}(d f X, d f Y)=\bar{b}(X, Y)-$ вторая фундаментальная форма гиперповерхности $\bar{M}, \bar{\nabla}-$ связность Леви-Чевита метрики $\bar{g}, \bar{n}$ - орт нормали гиперповерхности $\bar{M}$. Тогда [3]

$$
\partial_{X} d f Y-d f \bar{\nabla}_{X} Y=\bar{b}(X, Y) \bar{n} .
$$


Имеем

$$
\begin{aligned}
\partial_{X} \partial_{Y} d f Z-d f \bar{\nabla}_{X} \bar{\nabla}_{Y} Z-\bar{b}\left(X, \bar{\nabla}_{Y} Z\right) \bar{n} & =X \bar{b}(Y, Z) \bar{n}+\bar{b}(Y, Z) \partial_{X} \bar{n} \\
\partial_{Y} \partial_{X} d f Z-d f \bar{\nabla}_{Y} \bar{\nabla}_{X} Z-\bar{b}\left(Y, \bar{\nabla}_{X} Z\right) \bar{n} & =Y \bar{b}(X, Z) \bar{n}+\bar{b}(X, Z) \partial_{Y} \bar{n} \\
\partial_{[X, Y]} d f Z-d f \bar{\nabla}_{[X, Y]} Z & =\bar{b}([X, Y], Z) \bar{n}
\end{aligned}
$$

Так как

$$
\partial_{X} \partial_{Y} d f Z-\partial_{Y} \partial_{X} d f Z-\partial_{[X, Y]} d f Z=0
$$

имеем

$$
d f \bar{R}(X, Y) Z=\left(\left(\bar{\nabla}_{Y} \bar{b}\right)(X, Z)-\left(\bar{\nabla}_{X} \bar{b}\right)(Y, Z)\right) \bar{n}+\bar{b}(X, Z) \partial_{Y} \bar{n}-\bar{b}(Y, Z) \partial_{X} \bar{n}
$$

где $\left(\bar{\nabla}_{X} \bar{b}\right)(Y, Z)=X \bar{b}(Y, Z)-\bar{b}\left(\bar{\nabla}_{X} Y, Z\right)-\bar{b}\left(Y, \bar{\nabla}_{X} Z\right)-$ ковариантная производная поля $\bar{b}$ в связности $\bar{\nabla}, \bar{R}(X, Y) Z=\bar{\nabla}_{X} \bar{\nabla}_{Y} Z-\bar{\nabla}_{Y} \bar{\nabla}_{X} Z-\bar{\nabla}_{[X, Y]} Z-$ тензор кривизны связности $\bar{\nabla}$.

Так как $d f \bar{R}(X, Y) Z, \partial_{X} \bar{n} \in T \bar{M}$, получим

$$
\begin{aligned}
d f \bar{R}(X, Y) Z & =\bar{b}(X, Z) \partial_{Y} \bar{n}-\bar{b}(Y, Z) \partial_{X} \bar{n} \\
\left(\bar{\nabla}_{Y} \bar{b}\right)(X, Z) & =\left(\bar{\nabla}_{X} \bar{b}\right)(Y, Z) .
\end{aligned}
$$

2. Доказательство теоремы 1. По условию теоремы $\bar{R}=R$. Используя $(2),(5)$, получим

$$
b(Y, Z) d f A X-b(X, Z) d f A Y=\bar{b}(X, Y) \partial_{Y} \bar{n}-\bar{b}(Y, Z) \partial_{X} \bar{n} .
$$

Пусть $X_{i}, i=1, \ldots, n-1,-$ орты главных направлений гиперповерхности $M, k_{i}$ - главные кривизны. Тогда $A X_{i}=k_{i} X_{i}$. Полагаем $\partial_{X_{i}} \bar{n}=-\bar{A} d f X_{i}$ и при $n>3$ рассмотрим

$$
\begin{aligned}
k_{i} k_{j} d f X_{j} & =\bar{b}_{i i} \bar{A} d f X_{j}-\bar{b}_{i j} \bar{A} d f X_{i}, & i \neq j, \\
k_{\alpha} k_{j} d f X_{j} & =\bar{b}_{\alpha \alpha} \bar{A} d f X_{j}-\bar{b}_{\alpha j} \bar{A} d f X_{i}, & \alpha \neq i, j
\end{aligned}
$$

Так как $k_{i} k_{\alpha} \neq 0$, домножим первое равенство на $k_{\alpha}$, а второе на $k_{i}$ и отнимем. Получим

$$
\left(\bar{b}_{i i} k_{\alpha}-\bar{b}_{\alpha \alpha} k_{i}\right) \bar{A} d f X_{j}-\bar{b}_{i j} k_{\alpha} \bar{A} d f X_{i}+\bar{b}_{\alpha j} \bar{A} d f X_{\alpha}=0 .
$$

Так как $d f X_{i}, d f X_{j}, d f X_{\alpha}$ линейно независимые и $\operatorname{det} \bar{A} \neq 0$, получим

$$
\bar{b}_{i i} k_{\alpha}-\bar{b}_{\alpha \alpha} k_{i}=0, \quad \bar{b}_{i j}=0, \quad \bar{b}_{i \alpha}=0 .
$$

Итак,

$$
k_{i} k_{j} d f X_{j}=\bar{b}_{i i} \bar{A} d f X_{j} .
$$

Таким образом, линии кривизны гиперповерхности $M$ при отображении $f: M \rightarrow \bar{M}$ перейдут в линии кривизны гиперповерхности $\bar{M}$.

Положим $\bar{A} d f X_{i}=\bar{k}_{i} d f X_{i}$. Имеем

$$
\bar{b}_{i i}=\left\langle\bar{A} d f X_{i}, d f X_{i}\right\rangle=\bar{k}_{i} \bar{g}_{i i} .
$$

В силу (7), (8) имеем

$$
k_{i} k_{j}=\bar{k}_{i} \bar{k}_{j} \bar{g}_{i i} .
$$

Так как $n>3$, рассмотрим $k_{i} k_{\alpha}=\bar{k}_{i} \bar{k}_{\alpha} \bar{g}_{i i}, \alpha \neq j$. Отсюда $k_{j} / \bar{k}_{j}=k_{\alpha} / \bar{k}_{\alpha}$. Положим $k_{j} / \bar{k}_{j}=t$. Из (8), (9) имеем $\bar{g}_{i i}=t^{2}, \bar{b}_{i i}=t k_{i}=t b_{i i}$. Так как $X_{i}$ - орты главных направлений гиперповерхности $M$ и $d f X_{i}$ - главные направления гиперповерхности $\bar{M}$, то матрица $g$ в базисе $X_{i}$, единичная, а матрица $\bar{b}$ диагональная. Следовательно, имеем

$$
\bar{g}=t^{2} g, \quad \bar{b}=t b .
$$


Докажем, что $t=$ const. Диффференцируем равенство $\bar{b}(Y, Z)=t b(Y, Z)$ :

$$
\begin{aligned}
& \left(\bar{\nabla}_{X} \bar{b}\right)(Y, Z)+\bar{b}\left(\bar{\nabla}_{X} Y, Z\right)+\bar{b}\left(Y, \bar{\nabla}_{X} Z\right) \\
& \quad=(X t) b(Y, Z)+t\left(\nabla_{X} b\right)(Y, Z)+t b\left(\nabla_{X} Y, Z\right)+t b\left(Y, \nabla_{X} Z\right) .
\end{aligned}
$$

Так как отображение $f$ конформное, то [4, с. 84]

$$
\begin{aligned}
& \bar{\nabla}_{X} Y=\nabla_{X} Y+\omega(X) Y+\omega(Y) X-g(X, Y) U, \\
& \omega(X)=\frac{X t}{t}, \quad \omega(X)=g(X, U), \quad X, Y \in \chi(M) .
\end{aligned}
$$

Имеем

$$
\begin{aligned}
& \left(\bar{\nabla}_{X} \bar{b}\right)(Y, Z)+t \omega(Y) b(X, Z)-t g(X, Y) b(U, Z) \\
& \quad+t \omega(X) b(Y, Z)+t \omega(Z) b(Y, X)-t g(X, Z) b(Y, U)=t\left(\nabla_{X} b\right)(Y, Z) .
\end{aligned}
$$

Используем равенства (3), (6):

$$
g(X, Z) b(Y, U)-g(Y, Z) b(X, U)=0 .
$$

Полагая $X=Z=X_{i}, Y=X_{j}$, имеем $k_{j} \omega\left(X_{j}\right)=0, \omega\left(X_{j}\right)=0, X_{j} t=0, j=1, \ldots, n-1$, $t=$ const.

3. Доказательство теоремы 2. По теореме Бонее [1, с. 51] квадратичные формы $\bar{g}, \bar{b}$ определяют гиперповерхность $\bar{M}$ вплоть до положения в $E^{n}$, а гиперповерхность, определяемая уравнением $\bar{r}=t r$, имеет те же квадратичные формы. Следовательно, гиперповерхность $\bar{M}$ получена из гиперповерхности $M$ с помощью гомотетии и изометрии в $E^{n}$.

В частности, при изометрии $f$ имеем $t^{2}=1$ и имеет место теорема о жесткости для гиперповерхности в $E^{n}, n>3$ (см. [1, с. 47$\left.]\right)$.

\section{СПИСОК ЦИТИРОВАННОЙ ЛИТЕРАТУРЫ}

1. Кобаяси Ш., Номидзу К. Основы дифференциальной геометрии. Т. 2. М.: Мир, 1981. 2. Nomizu K., Yano K. // Math. Z. 1967. V. 97. P. 29-37. 3. Чешкова М. А. // Изв. Алтайского гос. ун-та, Барнаул. 1996. №1. С. 7-9. 4. Бессе А. Многообразия Эйнштейна. М.: Мир, 1990. 